\title{
Front Matter: Volume 9446
}

, "Front Matter: Volume 9446," Proc. SPIE 9446, Ninth International Symposium on Precision Engineering Measurement and Instrumentation, 944601 (6 March 2015); doi: 10.1117/12.2189854

SDIE Event: International Symposium on Precision Engineering Measurement and SPIE. Instrumentation, 2014, Changsha/Zhangjiajie, China 


\title{
PROCEEDINGS OF SPIE
}

\section{Ninth International Symposium on Precision Engineering Measurements and Instrumentation}

\author{
Jiubin Tan \\ Xianfang Wen \\ Editors \\ 8-10 August 2014 \\ Changsha, China
}

Organized by

International Committee on Measurements and Instrumentation - Instrumentation Committee of CSM (China) • Harbin Institute of Technology (China) • National University of Defense Technology (China)

Sponsored by

International Committee on Measurements and Instrumentation - National Natural Science Foundation of China (China) • Chinese Society for Measurement (China) • China Instrument and Control Society (China)

Cooperating Organizations

SPIE • Beijing Information Science and Technology University (China) • Hefei University of Technology (China)

Published by

SPIE 
The papers included in this volume were part of the technical conference cited on the cover and title page. Papers were selected and subject to review by the editors and conference program committee. Some conference presentations may not be available for publication. The papers published in these proceedings reflect the work and thoughts of the authors and are published herein as submitted. The publisher is not responsible for the validity of the information or for any outcomes resulting from reliance thereon.

Please use the following format to cite material from this book:

Author(s), "Title of Paper," in Ninth International Symposium on Precision Engineering Measurement and Instrumentation, edited by Jiubin Tan, Xianfang Wen, Proceedings of SPIE Vol. 9446 (SPIE, Bellingham, WA, 2015) Article CID Number.

ISSN: 0277-786X

ISBN: 9781628415612

Published by

SPIE

P.O. Box 10, Bellingham, Washington 98227-0010 USA

Telephone +1 3606763290 (Pacific Time) · Fax +1 3606471445

SPIE.org

Copyright (C) 2015, Society of Photo-Optical Instrumentation Engineers.

Copying of material in this book for internal or personal use, or for the internal or personal use of specific clients, beyond the fair use provisions granted by the U.S. Copyright Law is authorized by SPIE subject to payment of copying fees. The Transactional Reporting Service base fee for this volume is $\$ 18.00$ per article (or portion thereof), which should be paid directly to the Copyright Clearance Center (CCC), 222 Rosewood Drive, Danvers, MA 01923. Payment may also be made electronically through CCC Online at copyright.com. Other copying for republication, resale, advertising or promotion, or any form of systematic or multiple reproduction of any material in this book is prohibited except with permission in writing from the publisher. The CCC fee code is 0277-786X/15/\$18.00.

Printed in the United States of America.

Publication of record for individual papers is online in the SPIE Digital Library.

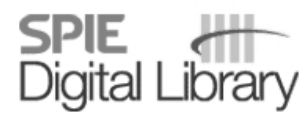

SPIEDigitallibrary.org

Paper Numbering: Proceedings of SPIE follow an e-First publication model, with papers published first online and then in print. Papers are published as they are submitted and meet publication criteria. A unique citation identifier (CID) number is assigned to each article at the time of the first publication. Utilization of CIDs allows articles to be fully citable as soon as they are published online, and connects the same identifier to all online, print, and electronic versions of the publication. SPIE uses a six-digit CID article numbering system in which:

- The first four digits correspond to the SPIE volume number.

- The last two digits indicate publication order within the volume using a Base 36 numbering

system employing both numerals and letters. These two-number sets start with 00, 01, 02, 03, 04, $05,06,07,08,09,0 A, 0 B \ldots 0 Z$, followed by 10-1Z, 20-2Z, etc.

The CID Number appears on each page of the manuscript. The complete citation is used on the first page, and an abbreviated version on subsequent pages. 


\title{
Contents
}

\author{
xiii Authors \\ xix Conference Committee \\ xxv Symposium Welcome \\ xxvii Introduction
}

\section{Part One}

NINTH INTERNATIONAL SYMPOSIUM ON PRECISION ENGINEERING MEASUREMENT AND INSTRUMENTATION

944602 Grating encoder for wide range three-axis displacement measurement [9446-1]

944603 Analysis of the rail's settlement on the measurement of the diameter of wheel-set [9446-5]

944604 Recovering fNIRS brain signals: physiological interference suppression with independent component analysis [9446-7]

944605 Design of CCD driver for quartz horizontal pendulum tiltmeter based on CPLD [9446-8]

944606 Design of handwriting drawing board based on common copper clad laminate [9446-10]

944607 Implementation of real-time displacement precision measurement technology for the sinusoidal phase-shifting laser self-mixing interferometer [9446-14]

944608 Correction of NIM-3A absolute gravimeter for self-attraction effect [9446-16]

944609 Online measurement for geometrical parameters based on 2D laser sensor [9446-17]

9446 0A Application of harmonic analysis method based on two-dimensional Fourier transform to flatness error sampling [9446-18]

$9446 \mathrm{OB} \quad$ Failure analysis of energy storage spring in automobile composite brake chamber [9446-19]

9446 OD Modeling of terahertz metamaterial-sensors for simulation based on effect of resonance induction [9446-21]

9446 OE Effect of self-vibration on accuracy of free-fall absolute gravity measurement with laser interferometer [9446-23]

9446 OF Extension of light transmission distance of single core fiber with a micro axicon fixed at fiber end [9446-31] 
9446 OG Compressive sensing image fusion based on blended multi-resolution analysis [9446-34]

$9446 \mathrm{OH} \quad$ Uncertainty evaluation for field experimental standard of vehicle speed-measuring devices in actual traffic [9446-35]

9446 Ol Design of Pound-Drever-Hall laser frequency stabilization system without phase shifter [9446-36]

9446 0J Measurement of the spectral characteristics and color parameters of flat objects [9446-37]

9446 OK The system of blade's shape measuring [9446-39]

9446 OL Multipurpose optic-electronic autocollimators for measuring deformations of the axle with a millimeter wave range radiotelescope (Invited Paper) [9446-40]

$94460 \mathrm{M}$ Optic-electronic systems for measurement a position of radio-telescope components [9446-41]

9446 ON A new suspension structure of micro/nano probe [9446-43]

944600 Calibration of GPS based high accuracy speed meter for vehicles [9446-47]

9446 OP Study on the detection of a cylindrical surface-breaking bore-hole reflector based on laser ultrasonic technology [9446-49]

9446 OQ Measurement of size error in industrial CT system with Calotte cube [9446-50]

9446 OR On-orbit calibration of space camera based on stellar image correspondences [9446-52]

9446 OS Modeling and active vibration control of six-DOF manipulator through $\mu$-synthesis with parameter uncertainties [9446-53]

9446 OT Reflective off-axis point-diffraction interferometer based on Michelson architecture [9446-54]

9446 OU Improved SIFT descriptor applied to stereo image matching [9446-56]

9446 OV Phase compensation with fiber optic surface profile acquisition and reconstruction system [9446-57]

9446 OW Establishment of theoretical model and experimental equipment for researching on carbon contamination of EUV multi-layer mirror [9446-58]

9446 OX Common mode EMI prediction and research in induction motor for electric vehicles [9446-59]

9446 OY Super-resolution imaging based on virtual Airy spot (Invited Paper) [9446-62]

$94460 Z$ Design of the digital quartz horizontal pendulum tiltmeter based on CCD [9446-63]

944610 Wireless communication bandpass optical window with double-layer hexagon aperture FSS array [9446-64] 

944611 Precision measurement of squareness of large rectangular square [9446-66]
944612 Design of extensible meteorological data acquisition system based on FPGA [9446-68]
944613 Application of coordinate transform on ball plate calibration [9446-69]
944614 Abnormal events detection in crowded scenes by trajectory cluster [9446-70]
944615 Design and implementation of embedded ion mobility spectrometry instrument based on SOPC [9446-72]
944616 An excitation signal source with anti-interference ability for eddy current testing [9446-73]
944617 A simple fiber optic humidity sensor based on water-absorption characteristic of CAB [9446-75]
944618 Research on cloud-based remote measurement and analysis system [9446-76]
944619 A new method for determining the Tikhonov regularization parameter of load identification [9446-77]

9446 1A GNSS software receiver sampling noise and clock jitter performance and impact analysis [9446-78]

$94461 \mathrm{~B} \quad$ Conversion of infrared grey-level image into temperature field by polynomial curve fitting [9446-80]

9446 1C Non-contact measurement of rotation angle with solo camera [9446-81]

9446 1D Position and orientation measurement during Lunar Rover movement test [9446-82]

9446 1E Finite element fatigue analysis of rectangular clutch spring of automatic slack adjuster [9446-83]

9446 IF Design of transmission error signal generator system based on FPGA and ARM [9446-85]

9446 IG High precision fabrication of antennas and sensors [9446-86]

$94461 \mathrm{H} \quad$ Optical fiber waist-enlarged bitaper-based Michelson interferometric humidity sensor [9446-87]

$944611 \quad$ Study on the station-moving measurement technology in the flatness measurement of large annular planes with a Laser Tracker [9446-88]

$94461 \mathrm{~J} \quad$ Small sample analysis of vision measurement error [9446-89]

$94461 \mathrm{~K} \quad$ Ultraviolet communication system based on BPSK subcarrier intensity modulation [9446-91]

9446 IL Simulation on measurement of five-DOF motion errors of high precision spindle with cylindrical capacitive sensor [9446-92] 
9446 1M Low-noise front-end electronics for detection of intermediate-frequency weak light signals [9446-93]

9446 iN Double-grating diffraction interferometric stylus probing system for surface profiling and roughness measurement [9446-94]

944610 Absolute distance measurement by spectral interferometry through wavelet transform with frequency comb [9446-99]

9446 IP Measurement time interval based on FPGA in NIM-3 absolute gravimeter [9446-100]

$94461 Q$ Optimization of the signal processing in frequency modulated continuous wave laser ranging system [9446-101]

9446 IR Improvement spatial resolution of frequency modulated continuous wave laser ranging system by splicing equal optical frequency interval sampled signal [9446-102]

9446 is Nonlinear analysis of cylindrical capacitive sensor used for measuring high precision spindle rotation errors [9446-103]

9446 IT Design of a data acquisition system of articulated arm coordinate measuring machines [9446-104]

$94461 \mathrm{U}$ Non-contact measurement for profile of different diameter micro/mini holes with capacitance sensor [9446-106]

9446 IV Analysis of Raman spectra of GeAsSe glass using different peak-fitting method [9446-108]

9446 IW A precision press-fit instrument for assembling small parts [9446-110]

9446 1X Detection of arc fault based on frequency constrained independent component analysis [9446-111]

9446 IY Research on the relationship between the curvature and the sensitivity of curved PVDF sensor [9446-113]

944612 Optimal design of a touch trigger probe [9446-116]

944620 On chip micro stress test circuit for miniature component [9446-117]

944621 Measurement of centering error for probe of swing arm profilometer using a spectral confocal sensor [9446-121]

944622 Front end design of smartphone-based mobile health [9446-122]

944623 Surface profile measurement of microstructures with steep slopes by sample-titling strategy [9446-123]

944624 Comparison between angle interferometer and angle encoder during calibration of autocollimator [9446-125] 
944625 Signal processing for single grating displacement measurement based on $3 \times 3$ coupler [9446-128]

944626 A new method for generation of non-diffraction grating structured light with phase shift [9446-131]

944627 Measurements of locomotive wheels using one-dimensional laser displacement sensor and eddy sensors [9446-132]

944628 Design of transmitter and receiver for experimental blue-green laser communication system [9446-137]

944629 Indirect measurement of machine tool motion axis error with single laser tracker [9446-138]

9446 2A Implementation of total focusing method for phased array ultrasonic imaging on FPGA [9446-140]

9446 2B Development of a scanning touch probe with 5-axis measuring functions [9446-141]

9446 2C Study on precision spatial measurement network of EAST [9446-142]

9446 2D Theoretical study and experimental verification on calculation of bearing capacity of aerostatic restrictor system with a gas-impedance model [9446-144]

$94462 \mathrm{E}$ Complex shape product tolerance and accuracy control method for virtual assembly [9446-146]

$94462 F \quad$ Improved LMD algorithm based on extraction of extrema of envelope curve [9446-147]

94462 2 Design and development of measuring device for beam pointing and positional errors in multi-axes laser interferometric systems [9446-150]

$94462 \mathrm{H} \quad$ Relaxation matching algorithm for moving photogrammetry [9446-154]

944621 Current ways and means for reduction or elimination of periodic nonlinearity in heterodyne interferometer [9446-158]

$94462 \mathrm{~J}$ Simulation analysis of position error of parabolic trough concentrator mirror installation [9446-159]

$94462 \mathrm{~K}$ Control methods of improving tracking precision [9446-160]

$94462 \mathrm{~L} \quad$ Alignment methods for partial compensating lens of aspheric testing in a non-null interferometer [9446-163]

$94462 \mathrm{M}$ Multiple-grating self-correcting algorithm for processed mark measurement error [9446-164]

$94462 \mathrm{~N} \quad$ A high-resolution detecting system based on machine vision for defects on large aperture and super-smooth surface (Invited Paper) [9446-167] 


\section{Part Two}

944620 Application of deadbeat control with constraint and non-ripple in precision rapid displacement system [9446-168]

9446 2P Vision-based on-machine measurement for CNC machine tool [9446-169]

$94462 \mathrm{E} \quad$ Error mechanism analyses of an ultra-precision stage for high speed scan motion over a large stroke [9446-170]

$94462 \mathrm{R}$ Performance analysis and experiment validation of a pneumatic vibration isolator [9446-172]

944625 Precisely connected and calculated algorithm of punctate scratches in the super-smooth surface defects evaluation system [9446-173]

$94462 \mathrm{~T} \quad$ Laser confocal measurement system for curvature radius of lenses based on grating ruler [9446-174]

$94462 \mathrm{U}$ Improving signal-to-noise ratio and reducing noise equivalent radiance of electro-optical systems sensor by binning image pixels [9446-175]

$94462 \mathrm{~V}$ Accuracy analysis of phase retrieval using Fourier transform method [9446-176]

9446 2W Analytical beam-width characteristics of distorted cat-eye reflected beam [9446-177]

$94462 X \quad$ Multifocal axial confocal microscopic scanning with a phase-only liquid crystal spatial light modulator [9446-178]

$94462 Y \quad$ The least square optimization in image mosaic [9446-179]

944630 Experiment study on the characteristics of two-dimensional line scale working standard [9446-181]

944631 Study on controllable LC-micro blazed grating beam deflector in free space [9446-185]

944632 The detection of wheel-flats based on fiber optic Bragg grating array [9446-186]

944633 Calibration of a high precision rotary table [9446-187]

944634 Altazimuth mount based dynamic calibration method for GNSS attitude measurement [9446-188]

944635 Ranging algorithm based on process measurement for low-altitude radio fuzes [9446-189]

944636 Development of a high accurate gear measuring machine based on laser interferometry [9446-190]

944637 Calibration of industrial CT using two forest-balls [9446-191]

944638 Characteristics of coated long-period fiber grating based on mode transition and dualpeak resonance [9446-193] 
944639 Static and dynamic property experiments of giant magnetostrictive material-fiber Bragg grating magnetic field sensors [9446-194]

9446 3A Development of ultra-precision centering and leveling turntable using aerostatic bearing technology [9446-195]

$94463 \mathrm{~B} \quad$ A voice coil motor based measuring force control system for tactile scanning profiler [9446-199]

$94463 \mathrm{C}$ A vertical scanning positioning system with large range and nanometer resolution for optical profiler [9446-200]

9446 3D Image inpainting for the differential confocal microscope [9446-201]

9446 3E Distributed compressive sensing of light field [9446-202]

$94463 \mathrm{~F} \quad$ Experimental study on absolute measurement of spherical surfaces with shift-rotation method based on Zernike polynomials [9446-203]

$94463 \mathrm{G}$ Coarse-fine vertical scanning based optical profiler for structured surface measurement with large step height [9446-205]

9446 3H Data communication between Panasonic PLC and PC using SerialPort control in C\#.NET environment [9446-206]

944631 Design and development of 80 meters laser interferometric measurement standard device [9446-207]

$94463 \mathrm{~J}$ Investigation of hidden diffuse surfaces using phase-shifting endoscopic digital speckle pattern interferometry [9446-208]

9446 3K Stitching interferometry for asphero-diffractive surface [9446-210]

9446 3L 3D reconstruction for sinusoidal motion based on different feature detection algorithms [9446-212]

$94463 \mathrm{M}$ Vibration studies of simply supported beam based on binocular stereo vision [9446-214]

$94463 \mathrm{~N}$ Multifunction surface measurement system based on focusing optical stylus interference and confocal image [9446-218]

944630 Anti-jamming capability of pseudorandom noise code ranging systems under narrowband interference [9446-220]

$94463 \mathrm{P}$ The propagation of manufacture uncertainty to dynamic measurement [9446-221]

$94463 Q$ Theoretical analysis of harmonic suppression in multi-step error separation technique [9446-222]

$94463 R \quad$ Influence of diffraction effect on measurements of absolute gravity [9446-223] 
944635 Analysis of pneumatic hammer in rectangular aerostatic thrust bearing with groove [9446-225]

9446 3T Implementation of primary low-g shock standard for laser interferometry [9446-226]

$94463 \mathrm{U}$ Improvement of spatial resolution in confocal microscope with shifted-focus phase filter [9446-227]

9446 3V Elimination of Abbe error method of large-scale laser comparator [9446-229]

9446 3W Multimode vibration damping as a result of piezoelectric energy harvesting [9446-230]

$94463 \mathrm{X}$ Study on formation and transformation of the optical nonlinearity harmonics in the heterodyne laser interferometer [9446-231]

$94463 Y$ Equivalent common path method in large-scale laser comparator [9446-234]

$944632 \quad$ Beam shaping with vortex beam generated by liquid crystal spatial light modulator [9446-235]

944640 Refractive index of air for interferometric length measurements (Invited Paper) [9446-236]

944641 Effects of dynamic characters of the macro-micro fast coupling system in long stroke system [9446-237]

944642 Analyzing time walk error of leading trailing edge CFD of timing discrimination for Gaussian and Rayleigh distribution waveform [9446-239]

944643 Adjusting and positioning method with high displacement resolution for large-load worktable based on the invariable restoring force [9446-240]

944644 Research on measurement of lateral dimension based on digital micromirror device [9446-241]

944645 Development and validation of a lateral MREs isolator [9446-243]

944646 Effect of reflector angle on the performance of a fiber bundle distance sensor with single mode illumination [9446-244]

944647 High-speed measurement of nozzle swing angle of rocket engine based on monocular vision [9446-245]

944648 Multi-functional hinge equipped with a magneto-rheological rotary damper for solar array deployment system [9446-246]

944649 A method of gear defect intelligent detection based on transmission noise [9446-247]

$94464 \mathrm{~A}$ Working-point control technique for the homodyne interferometry in hydrophone calibration (Invited Paper) [9446-249]

9446 4B Design and implementation of an illumination device for optical inspection of defects in glass substrates [9446-250] 
9446 4C Discuss on traceability method of light-scattering airborne particle counter's counting performance [9446-252]

$94464 \mathrm{D}$ Analysis of the effect anisotropic retards collimation turns on polarization and energy radiation parameters [9446-253]

$94464 \mathrm{E} \quad$ Calibration of rotary table by whole combination measuring method [9446-255]

$94464 \mathrm{~F} \quad$ Investigating of precision measurement on ultrasonic flow [9446-256]

9446 4G Generation of stainless steel superhydrophobic surfaces using WEDM technique [9446-257]

$94464 \mathrm{l} \quad$ Portable and modularized fluorometer based on optical fiber [9446-261]

9446 4J A displacement measuring system based on grating double diffraction [9446-262]

$94464 \mathrm{~K}$ Simulation research on ATP system of airborne laser communication [9446-263]

$94464 \mathrm{~L} \quad$ Modeling and simulation of continuous wave velocity radar based on third-order DPLL [9446-264]

$94464 \mathrm{M}$ Recent advances in absolute distance measurements using femtosecond light pulses (Invited Paper) [9446-266]

$94464 \mathrm{~N} \quad$ Absolute distance measurement using frequency-comb-referenced four-wavelength interferometry [9446-267]

944640 Communication design for multi-boards based on VME bus [9446-268]

$94464 \mathrm{P} \quad$ Parameter estimation of an air-bearing suspended test table [9446-269]

$94464 Q \quad$ Four-wheel alignment based on computer vision [9446-271]

$94464 \mathrm{R} \quad$ Measurement of duration of AC transient signal waveform using Hilbert transform and least square method [9446-272]

9446 4S Detection of sub-pixel chessboard corners based on gray symmetry factor [9446-276]

$94464 \mathrm{~T} \quad$ Research on dual-wavelength photometric method for micro liquid volume measurement [9446-282]

$94464 \mathrm{U}$ An improved PSO algorithm and its application on model identification of coarse-stage in lithography machine [9446-283]

$94464 \mathrm{~V}$ A novel method for measuring transit tilt error in laser trackers [9446-284]

9446 4W Research on PID controller with input shaping algorithm for linear motor [9446-286]

9446 4X Passive protective strategy for ultra-precision dual-stage [9446-287]

$94464 \mathrm{Y} \quad$ Cogging force rejection method of linear motor based on internal model principle [9446-288] 
$94464 Z$ Multi-view 3D measurement data registration based on encoding point spatial location and match [9446-289]

944650 Real time measurement for deformation of large expansion frame based on retro-reflective technique and vision method [9446-291]

944651 Measurement of manufacturing resolution for two photon polymerization structures with different manufacturing parameters [9446-292]

944652 Calibration of positional consistency between mechanical shaft and electronic boresight for radar antenna [9446-296]

944653 A new wideband interference suppression method for GNSS system [9446-300]

944654 Influence of measuring algorithm on shape accuracy in the compensating turning of high gradient thin-wall parts [9446-302]

944655 The precision measurement and assembly for miniature parts based on double machine vision systems (Invited Paper) [9446-303]

944656 Detection of wheel rim by immersion scan of phased array ultrasonic flaw testing [9446-304]

944657 Experimental study on imaging spectrometer focusing formula in orbit [9446-306]

944658 Stiffness modeling of flexible suspension structure for displacement measurement probing sensors [9446-307]

944659 Research and development of novel wireless digital capacitive displacement sensor [9446-310]

9446 5A A novel active suppression technology against thermal drift for ultra-precision spherical capacitive sensors [9446-311] 


\section{Authors}

Numbers in the index correspond to the last two digits of the six-digit citation identifier (CID) article numbering system used in Proceedings of SPIE. The first four digits reflect the volume number. Base 36 numbering is employed for the last two digits and indicates the order of articles within the volume. Numbers start with 00, 01, 02, 03, 04, 05, 06, 07, 08, 09, 0A, 0B...0Z, followed by 10-1Z, 20-2Z, etc.

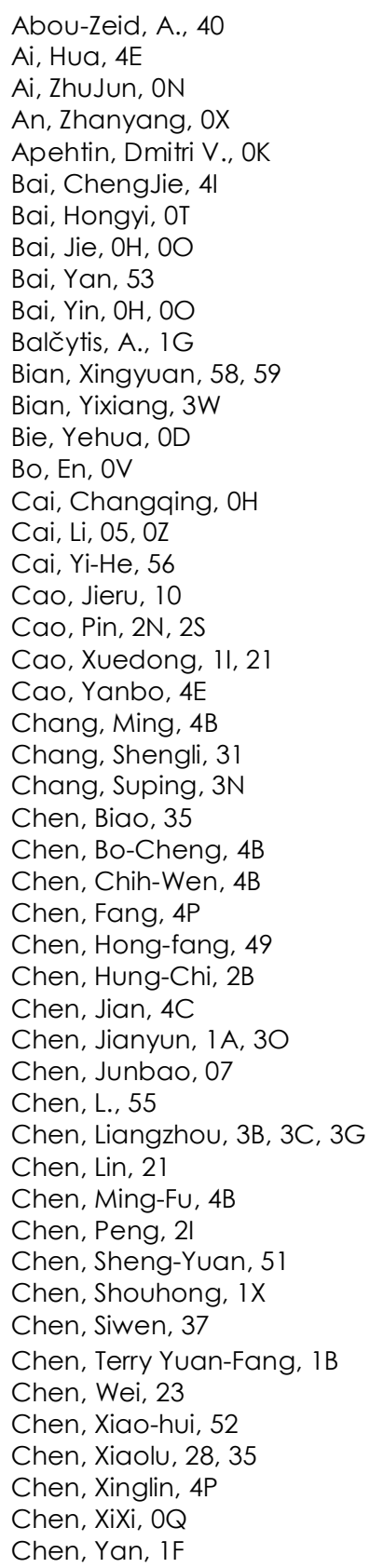

Chen, Yangjie, 2L, 2N, 2S

Chen, Yongzhi, $1 \mathrm{X}$

Chen, Yuanzheng, OF

Chen, Zhemin, $1 \mathrm{H}$

Chen, Zhenyu, $4 \mathrm{X}, 4 \mathrm{Y}$

Chen, Zhongzhu, 4S

Chen, Zichen, is

Cheng, Jia, $1 \mathrm{H}$

Cheng, Yang, 2D

Chertov, Aleksandr N., 0J

Chin, C., 04

Chu, Chih-Liang, 2B

Chu, ZhaoRui, ON

Chun, Byung Jae, 4M, 4N

Chung, Tien-Tung, 51

Cui, Chang-cai, 44

Cui, Haihua, $4 Z$

Cui, Jiwen, OF, 2M, 2O, 2Q, 3S, 41

Cui, Junning, OY, 58, 59, 5A

Deng, Huaxia, 3L, 3M, 3P

Deng, Xiangrui, 3I, 3V

Di, Yan, 4L

Ding, Guoping, 39

Ding, Xue-mei, 2l, 20

Dong, Chun, 4F

Dong, Lijun, 42

Dong, Mingli, 2J

Dong, Weijie, $1 Y$

Dong, Yibo, 4F

Dong, Yue, 4W

Du, Jianhao, 22

Du, Lei, $\mathrm{OH}, 0 \mathrm{O}$

Du, Zhengchun, 29

Du, Zhiguang, $1 \mathrm{M}$

Duan, Fajie, OV

Fan, Kuang-Chao, 1 Z

Fan, Tian-quan, 11

Fan, Wei, 44

Fan, Wenchao, 4W

Fan, Zhigang, 3K, 3X

Fan, Zongwei, 1L, 1S, 1U

Feng, Fan, OV

Feng, Jian, $1 \mathrm{Z}$

Feng, Jin-yang, 08, OE, 1P, 3R

Feng, Qibo, 03, 09, 27

Feng, Shengdong, 3B

Feng, XuZhe, 1A

Fu, Haijin, $3 X$

Fu, Jie, 45, 48 
Fu, Zhenxian, 4O, 4P, 4U, 4W, 4X

Gabalis, M., $1 G$

Gan, Jianghong, 26

Gan, Xiaochuan, 1C, 3H

Gan, Yu, $3 Z$

Gan, Yu-Lin, IV

Gao, Bin, 39

Gao, Guanbin, $1 T$

Gao, Ran, 11

Gao, Sitian, 37

Gao, Ting, 3H

Gao, Wei, 19

Gao, Wenzhi, 06

Gao, Xiao-rong, 2A, 32, 56

Gao, Yan, 3J

Gao, Yinhan, OX

Gao, Yue, $3 Z$

Gao, Zhiliang, 4C

Gao, Zhiqiang, 18, 22

Gong, Xuepeng, OW

Gorbachev, Alexey A., OK

Gorbunova, Elena V., OJ

Gu, Qing, 34

Gu, Song, 1K

Gu, Wei, 3Q, 43

Gu, Yongqi, 2C

Gu, Zhengtian, 38

Guan, Jian, 02

Guo, Changye, $4 Z$

Guo, Cheng, OY

Guo, Dongmei, 07

Guo, Jian-qiang, 2A, 32, 56

Guo, Lei, 2H, 3Z, 42

Guo, Lili, OT

Guo, Mian, 49

Guo, Ruipeng, OP

Guo, Tenghui, 43

Guo, Tiantai, 2F

Guo, ZhenYa, 4l

Han, Jiang, $2 \mathrm{P}$

Han, Lu, 59

Han, Tao, 53

Han, Yu, 10

Hao, Biao, 20

$\mathrm{Hao}, \mathrm{Hui}, 07$

$\mathrm{He}$, Hongtao, 09

He, Lingsong, 18, 22

$\mathrm{He}$, Mingzhao, 3l, 3V, 3Y

$\mathrm{He}$, Tao, 34

$\mathrm{He}$, Zhangqiang, 59

Hoi, Chi-Hou, 51

Hong, Ma, 4L

Hou, Maosheng, 3A, 3D

Hou, Xi, 3F

Hu, Guoxing, OP

Hu, Hong-bo, 3T

Hu, Jiacheng, 2D

$\mathrm{Hu}$, Pengbing, $1 \mathrm{H}$

Hu, Peng-cheng, 2G, 2 I

$\mathrm{Hu}$, Xiao-feng, OB, $1 \mathrm{E}$
Huang, Dongzhao, $4 Q$

Huang, Fu-gui, OA, 44

Huang, Hailong, $4 \mathrm{~K}$

Huang, Jingzhi, 3Q, 43

Huang, Tingting, OV

Huang, Xiangdong, 3U

Huang, Xuguang, 17

Huang, Yao, 24, 33, 36

Huang, Yubo, 23

Hyun, Sangwon, 4M

Jang, Yoon-Soo, 4M, 4N

$\mathrm{Ji}, \mathrm{An}, \mathrm{OD}$

Ji, Lin, 3A

Ji, Qizheng, 4C, 4F

Jia, Min-qiang, 11

Jiang, Fangliang, 4F

Jiang, Guodong, 16

Jiang, Kai, 50

Jiang, Li, 52

Jiang, Nan, 34

Jiang, ShouZhen, $4 \mathrm{I}$

Jiang, Wen-song, 1E

Jiang, Xiang-dong, 56

Jiang, Yanwei, 15

Jiang, Yong, 46

Jiao, Mingxing, 0

Jin, Hui, 57

Jin, Peng, 02

Jin, Yuanqiang, 2E

Jing, Hongwei, 21, 3F

Juodkazis, S., $1 \mathrm{G}$

Kang, Hyunjay, 4N

Kim, Seung-Woo, 4M, 4N

Kim, Young-Jin, 4M, 4N

Kong, Ming, $0 Q, 2 F$

Konyakhin, Alexey, OL

Konyakhin, Igor A., OL, OM

Korotaev, Valery $\mathrm{V}$., OJ, OK, 4D

Kuo, Ming-Hsuan, 1B

Lai, Kuan-Wen, 2B

Lan, Jinlong, 38

Lastovskaia, Elena A., OJ

Lei, Rui, 3E

Lei, Zili, 3C, 3G

Li, Chen, 2N, $2 S$

Li, Chun-jian, 08, 0E, 1P, 3R

Li, Dan, 1J

Li, Dongsheng, 2D

Li, Guang, 17

Li, Guoshui, 1H

Li, Jiafu, 2D

$\mathrm{Li}$, Jianshuang, 3I, 3V, 3Y

Li, Jie, 1 I

Li, Leilei, 58

Li, Lianfu, 3I, 3V

Li, Liangliang, 29

Li, LU, 2N, 2S

Li, Peng, 20

Li, Po, 16

Li, Qi, 37 


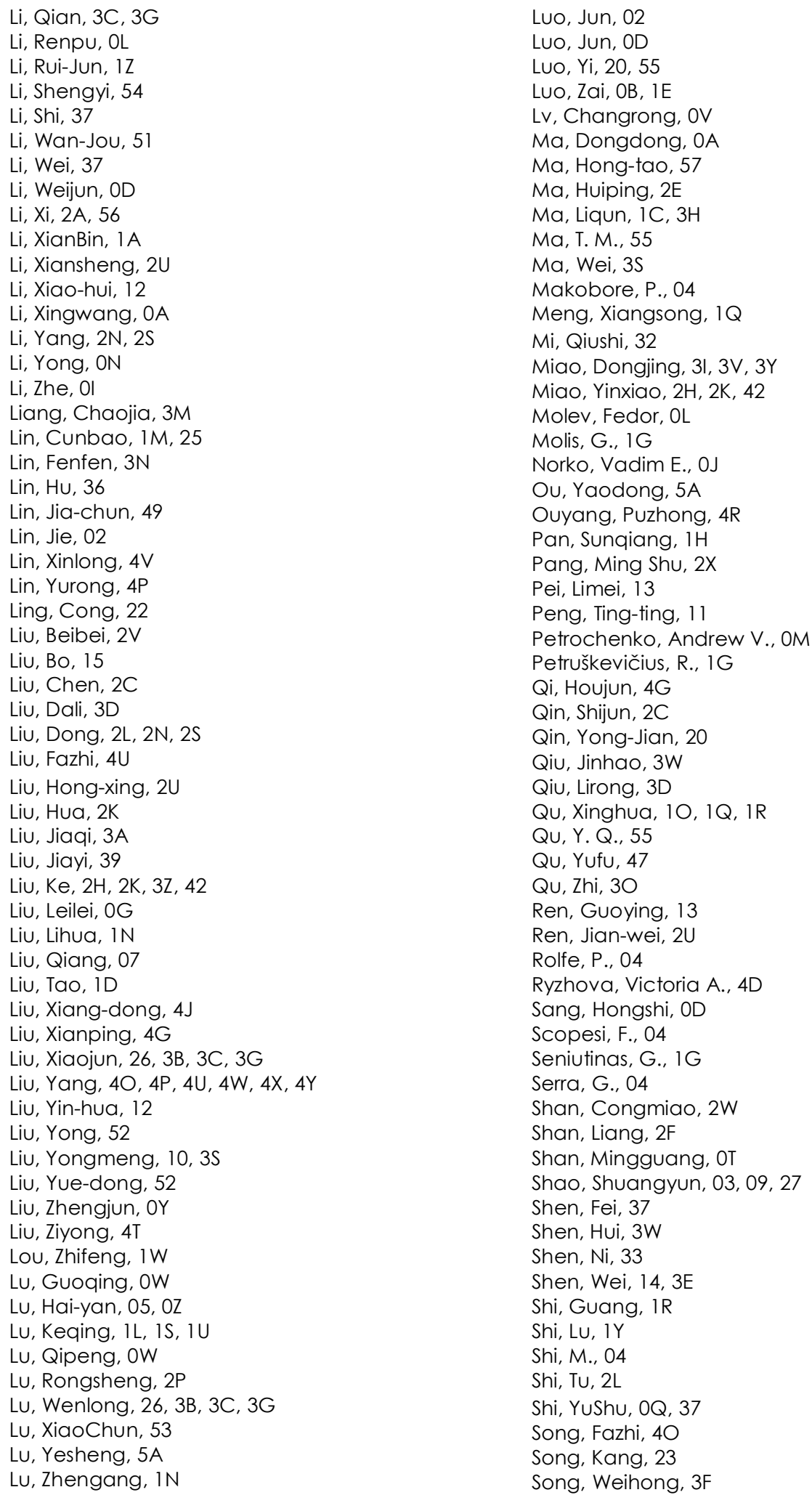


Song, Xiaoping, 37

Song, $\mathrm{Xu}, 37$

Song, Yuqian, $2 F$

SU, DUO-WU, 08, 0E, 1P, 3R

Su, Juan, 0

Su, Wei, 18

Sun, Anbin, $1 C$

Sun, Huayan, 2W

Sun, J., 04

Sun, Peng, 2J

Sun, Qiao, $\mathrm{OH}, 0 \mathrm{O}, 3 \mathrm{~T}$

Sun, Shaohua, 34

Sun, Shuanghua, 30

Sun, Tao, 43, 58, 59, 5A

Sun, Xiaolei, 28

Sun, Yanling, $3 \mathrm{~N}$

Sun, Zeng-yu, $3 Z$

Tan, Jianxiong, 03

Tan, Jiu-bin, OF, 10, 2I, 2M, 2O, 2Q, 2R, 3Q, 3S, 3X

Tang, Jie, $\mathrm{ON}$

Tang, Laiying, $1 \mathrm{D}$

Tang, Minghui, $4 \mathrm{Z}$

Tao, Ronghua, 35

Tao, Wei, 50

Tao, Yufeng, 07

Tian, GuiYun, OP

Tian, Guo-liang, 2J

Tian, Jiwei, 2T

Tian, Yanling, 4G

Tian, Yanrong, 3A

Tolochek, Nina S., OM

Tong, Lin, 4T

Tong, Ying, OG

Trushkina, Anna V., 4D

Urbonas, D., $1 G$

Valušis, G. ', $1 G$

Vaškevičius, K., $1 G$

Wan, Bile, ID

Wan, Min, OP

Wan, Zhi, $2 \mathrm{U}$

Wang, Bao Kai, $2 \mathrm{X}$

Wang, Can, 18

Wang, Chongyang, $3 \mathrm{U}$

Wang, DaoDang, $0 Q$

Wang, Dong-wei, 52

Wang, FuMin, $0 Q$

Wang, Guilin, 54

Wang, Guochao, 1M, 25, 4N

Wang, Haitao, OP

Wang, He Yan, 24, 30, 33, 36

Wang, HongTao, ON

Wang, Hongyuan, 06

Wang, Jian-lin, 3T

Wang, Jin, 53

Wang, Jintao, $4 \mathrm{~T}$

Wang, Jun, 2C

Wang, Jun, 2J

Wang, Jun, 3P

Wang, Juxian, OX

Wang, Lei, 2R, 4J
Wang, Lei, 3D

Wang, Li, IV

Wang, Longxiao, 3A

Wang, Ming, 07

Wang, Quanyang, 46

Wang, Shaokai, $2 Q$

Wang, Shitong, 2N, $2 S$

Wang, Shuai, ON

Wang, Tao, 54

Wang, Tianhao, $\mathrm{OX}$

Wang, Weibo, 3K

Wang, Weinong, 13

Wang, Wen, 1L, 1S, 1T, $1 \mathrm{U}$

Wang, Xiao-Dong, 1W, 20, 55

Wang, Xiu, 05, OZ

Wang, Yingjun, $2 \mathrm{~F}$

Wang, Yong, $1 \mathrm{~K}$

Wang, Yu, OA

Wang, Yuan, 06

Wang, Yun, 2T

Wang, Ze-yong, 2A, 32, 56

Wang, Zhongyu, $1 \mathrm{~J}$

Wei, Chunhua, 1M, 25

Wei, Hengzheng, 13

Wei, Kai, 4O, 4U

Wei, Peipei, $1 \mathrm{~N}$

Wei, Qing, OB

Wei, Zhongwei, 21

Wei, Zilong, OG

Wen, Dong, 28

Wen, Feng, 02

Wen, Mingfu, 48

Wen, Zhongpu, 43

Weng, Jinping, $4 Z$

Weng, Rui-Cian, 4B

Wickramasinghe, Y. A. B. D., 04

Wu, Fan, 3F

Wu, GuangYao, $1 \mathrm{~A}$

Wu, Hanzhong, 10

Wu, Jianwei, 41

Wu, Qun, OY

Wu, Shi-bin, 11, 3F

Wu, Shu-qing, 08, 0E, 1P, 3R

Wu, Sijin, 3J

Wu, Ting, $2 G$

Wu, Xiaoyan, 2V

Wu, Ying, OS

Wu, Zhaoyong, 29

Wu, Zhengzhong, 48

Xia, Lian, $2 \mathrm{P}$

Xia, Ruixue, 2P

Xiang, Kui, 1L, $1 S$

Xiang, Meng, $1 \mathrm{Z}$

Xiang, Xiaoyan, $3 \mathrm{U}$

Xiao, Fu, OV

Xie, Changsheng, OD

Xie, Shibin, $2 S$

Xie, Shibing, $2 \mathrm{~N}$

Xie, Tiebang, 3N

Xin, Liwei, 42 
Xin, M. Z., 55

Xing, Guangzhen, 4A

Xing, Junhong, OI

Xing, Zhi-Wei, 45

Xiong, Wei, OR, OU

Xiong, Weixing, 27

$X u, B i n, 23$

$X U, C h e n-j i e, 1 E$

$X U$, Jin-yi, 08, 1P, 3R

$X U$, Long, 26

$X u$, Min-er, 4J

$\mathrm{XU}$, Renhao, $1 \mathrm{X}$

$X \cup$, Suzhi, 31

$\mathrm{Xu}, \mathrm{TaO}, 28,35$

$\mathrm{XU}$, Wei, 17

$\mathrm{XU}$, Yang, $\mathrm{ON}$

$X U$, Yang, $1 \mathrm{~W}$

Xue, Changxi, $2 U$

Xue, Zi, 24, 30, 33, 36

Yan, Bi-xi, 2J

Yan, LU, 2N, 2S

Yan, Shuhua, 1M, 25

Yan, Siwen, 3K

Yang, C., 04

Yang, Guoliang, 36

Yang, Haijuan, 47

Yang, Huiping, $1 T$

Yang, Jianhong, $1 \mathrm{X}$

Yang, Jie, 15

Yang, Jie, 11

Yang, Jun, 30

Yang, Junbo, 31

Yang, Kai, $1 \mathrm{X}$

Yang, Kaiyu, OX

Yang, Lianxiang, 3J

Yang, Liu, 15

Yang, Ping, 4A

Yang, Tianbo, $4 Y$

Yang, Tianlong, 4Q, 4S

Yang, Xianming, $\mathrm{OP}$

Yang, Xiao, $3 G$

Yang, Yongying, 2L, 2N, 2S

Yang, Yong-yue, $2 Y$

Yang, Yuanyuan, $2 R$

Yang, Zaihua, 1D

Yang, Zhen-yu, 05, $0 Z$

Ye, Xin, $1 \mathrm{C}$

Yi, Wangmin, ID

You, Bo, IW

YU, Kaiping, OS, 19

YU, Liandong, 3L, 3M, 3P

Yu, Mei, OE, OO

Yu, Miao, 45, 48

Yu, Qing, 44

Yu, Yingjie, $2 V$

Yuan, Guibin, 2G

Yuan, Yong, 41

Yue, WeiWei, 41

Zeng, Dan, 14

Zeng, Luan, OR, OU
Zeng, Wei, OP

Zhai, Dongwei, 4F

Zhai, You, OR, OU

Zhan, Wei-wei, 05, 0 Z

Zhang, Biyun, 39

Zhang, Changfan, 18, 22

Zhang, Fengsheng, 3W

Zhang, Fumin, 10, 1Q, 1R

Zhang, Genwei, 15

Zhang, Hui-jun, 12

Zhang, Jian, 11

Zhang, Jianfeng, $1 \mathrm{H}$

Zhang, Jin, 3L, 3M, 3P

Zhang, Jingjing, 31

Zhang, L. F., 55

Zhang, Laixian, $2 \mathrm{~W}$

Zhang, Lei, $2 \mathrm{~L}$

Zhang, Lei, 4I

Zhang, Manshan, 3V

Zhang, Mengqian, 3K

Zhang, Min, 1L, 1S

Zhang, Peng, 3L

Zhang, Rencheng, IX

Zhang, Tao, $2 \mathrm{M}$

Zhang, Wen, 12

Zhang, Xiaoguang, 2E

Zhang, Xinyu, OD

Zhang, Xunbiao, 4C

Zhang, Y., 04

Zhang, Y. J., 04

Zhang, Yabin, OT

Zhang, Yifei, 2G

Zhang, Yu-dong, $2 Y$

Zhang, Yue, $\mathrm{OH}$

Zhang, Yue, $1 Y$

Zhang, Zhi-jiang, 14, 3E

Zhang, Zhonghua, 4R

Zhang, Zili, 4V

Zhao, Bo, 4J

Zhao, Dong-sheng, 11

Zhao, Hong, 4J

Zhao, Hui, 50

Zhao, Jiang, 15

Zhao, Jun, $0 Q, 2 F$

Zhao, Limin, $2 \mathrm{~N}$

Zhao, Lu-Jie, 45

Zhao, Meirong, OG

Zhao, Qiancheng, 4Q, 4S

Zhao, Quanke, 2A, 32

Zhao, Shiping, 23

Zhao, Shuling, 3N

Zhao, Weiqian, 2T, 3A, 3D

Zhao, Weirui, 2T

Zhao, Yafeng, 4S

Zhao, Yanzhong, 2W

Zhao, Yun, 49

Zhao, Zhimin, 4 Z

Zhao, Zhongyi, 4K

Zheng, Yi, 3C, 3G

Zheng, Yong, $1 \mathrm{~F}$ 
Zheng, Yonghui, 2W

Zheng, Yuanyang, 2C

Zhong, Zhi, OT, 46

Zhou, Fangyuan, 26

Zhou, Hai, 2E

Zhou, Hao, $1 \mathrm{Z}$

Zhou, Liping, 26, 3B

Zhou, Meng Jiao, $2 X$

Zhou, Nan, 2T

Zhou, Shifu, 14

Zhou, Tong, OF, 2R

Zhou, Weihu, 4V

Zhou, Wenjing, $2 \mathrm{~V}$

Zhou, Ying, 3E

Zhu, Chen, 4L

Zhu, Dengchao, 54

Zhu, Han, 4V

Zhu, Hongna, 32

Zhu, Jigui, $2 \mathrm{H}$

Zhu, Lianqing, 3J

Zhu, Liyan, $1 \mathrm{U}$

Zhu, Wen, 11

Zou, Li Min, 2X 


\title{
Conference Committee
}

\author{
Honorary Chairs
}

Guofan Jin, Tsinghua University (China)

Songlin Zhuang, University of Shanghai for Science and Technology (China)

Tongbao Li, Tongji University (China)

Zhonghua Zhang, National Institute of Metrology (China)

Jie Gao, Sichuan University (China)

Shenghua Ye, Tianjin University (China)

Zhu Li, Huazhong University of Science and Technology (China)

Ahmed Abou-zeid, Physikalisch-Technische Bundesanstalt (Germany)

Peter Rolfe, Università degli Studi di Genova (Italy)

Yetai Fei, Hefei University of Technology (China)

\section{Conference Chair}

Jiubin Tan, Harbin Institute of Technology (China)

\section{Conference Co-chairs}

Tony Wilson, University of Oxford (United Kingdom)

Harald Bosse, Physikalisch-Technische Bundesanstalt (Germany)

K. C. Fan, National Taiwan University (Taiwan, China)

Wei Gao, Tohoku University (Japan)

\section{Program Committee Chair}

Jiubin Tan, Harbin Institute of Technology (China)

Program Committee Co-chairs

Tony Wilson, University of Oxford (United Kingdom)

Harald Bosse, Physikalisch-Technische Bundesanstalt (Germany)

Min Gu, Swinburne University of Technology (Australia)

K. C. Fan, National Taiwan University (Taiwan, China)

Wei Gao, Tohoku University (Japan)

Seung-Woo Kim, Korea Advanced Institute of Science and

Technology (Korea, Republic of) 
Program Committee Members

Frank Härtig, Physikalisch-Technische Bundesanstalt (Germany)

Liangchi Zhang, University of New South Wales (Australia)

Shulian Zhang, Tsinghua University (China)

Igor A. Konyakhin, Saint-Petersburg State University of Information Technologies, Mechanics and Optics (Russia)

Alexander Poleshchuk, Institute of Automation and Electrometry, Russian Academy of Sciences (Russia)

Martin Booth, University of Oxford (United Kingdom)

Aiwen Ma, China Instrument and Control Society (China)

Youhua Wu, Chinese Society for Measurement (China)

Guobiao Wang, National Natural Science Foundation of China (China)

Norihiro Umeda, Tokyo University of Agriculture and Technology (Japan)

Richard Leach, National Physical Laboratory (United Kingdom)

Liang-Chia Chen, National Taipei University of Technology

(Taiwan, China)

Jens Flügge, Physikalisch-Technische Bundesanstalt (Germany)

Yusaku Fujii, Gunma University (Japan)

Ming Chang, Chung Yuan Christian University (Taiwan, China)

Xianping Liu, University of Warwick (United Kingdom)

Senyung Lee, National Cheng Kung University (Taiwan, China)

Otto Jusko, Physikalisch-Technische Bundesanstalt (Germany)

Jiwen Cui, Harbin Institute of Technology (China)

Chenggen Quan, National University of Singapore (Singapore)

Wenmei Hou, University Shanghai for Science and Technology (China)

Michael Krystek, Physikalisch-Technische Bundesanstalt (Germany)

Zengyao Lin, Industrial Technology Research Institute (Taiwan, China)

Gaoliang Dai, Physikalisch-Technische Bundesanstalt (Germany)

J.F. Song, National Institute of Standards and Technology

(United States)

K. Minoshima, National Metrology Institute of Japan (Japan)

Rongcing Lin, National Taiwan University (Taiwan, China)

Jian Liu, Harbin Institute of Technology (China)

\section{Organizing Committee Chair}

Yifan Dai, National University of Defense Technology (China)

\section{Organizing Committee Co-chairs}

Yongsheng Gao, University of Science and Technology (China)

Jun Yang, National University of Defense Technology (China)

Tianquan Fan, Institute of Optics and Electronics, Chinese Academy of Sciences (China) 
Xinghua Qu, Tianjin University (China)

Lijiang Zeng, Tsinghua University (China)

Jiaru Chu, University of Science and Technology of China (China)

Zili Zhou, Changcheng Institute of Metrology and Measurement (China)

Zhaoyao Shi, Beijing University of Technology (China)

Lianqing Zhu, Beijing Information Science and Technology University (China)

Zhenghui Zhu, China Academy of Launch Vehicle Technology (China)

\section{Organizing Committee Members}

Yongbin Zhou, National University of Defense Technology (China)

Shibin Wu, Institute of Optics and Electronic, the Chinese Academy of Science (China)

Yuchi Lin, Tianjin University (China)

Qibo Feng, Beijing Jiaotong University (China)

Dongsheng Li , China Jiliang University (China)

Yinhan Gao, Jilin University (China)

Junjie Guo, Xi' an Jiaotong University (China)

Donglin Peng, Chongqing University of Technology (China)

Jianhua Wang, Xi'an Technological University (China)

Yan Li, Tsinghua University (China)

Xiaogang Sun, Harbin Institute of Technology (China)

Linna Zhang, Zhengzhou University (China)

Xiangzhao Wang, Shanghai Institute of Optics and Fine Mechanics, Chinese Academy of Sciences (China)

Xiaoyang Yu, Harbin University of Science and Technology (China)

Guoyu Zhang, Changchun University of Science and Technology (China)

Yinxiao Miao, Beijing Aerospace Institute of Metrology and Measurement (China)

Yongying Yang, Zhejiang University (China)

Zhongyu Wang, Beihang University (China)

Qun Hao, Beijing Institute of Technology (China)

Dengxin Hua, Xi'an University of Technology (China)

Hui Zhao, Shanghai Jiaotong University (China)

Xiaodong Wang, Dalian University of Technology (China)

Junbi Liao, Sichuan University (China)

Yingjie Yu, Shanghai University (China)

Liandong Yu, Hefei University of Technology (China)

Shiyuan Liu, Huazhong University of Science and Technology (China)

Weihu Zhou, Academy of Opto-electronics, Chinese Academy of Sciences (China)

Yong Xu, Changcheng Institute of Metrology \& Measurement (China) Weiqian Zhao, Beijing Institute of Technology (China) 
Tiehua Ma, North University of China (China)

Zhiquan Li, Yanshan University (China)

Yongrui Zhao, China University of Petroleum (China)

Mingxing Jiao, Xi' an University of Technology (China)

Yanqiu Li, Beijing Institute of Technology (China)

Secretaries-General

Xianfang Wen, Harbin Institute of Technology (China)

Peng Jin, Harbin Institute of Technology (China)

Junning Cui, Harbin Institute of Technology (China)

Deputy Secretary-General

Jie Lin, Harbin Institute of Technology (China)

Secretaries

XingYuan Bian, Harbin Institute of Technology (China)

Ying Tan, Harbin Institute of Technology (China)

Meiqi Zhang, Harbin Institute of Technology (China)

Session Chairs

1 Instrumentation Theory and Methodology I

Michael Krystek, Physikalisch-Technische Bundesanstalt (Germany) Jiwen Cui, Harbin Institute of Technology (China)

2 Measurement for Precision and Ultra-Precision Machining

Gaoliang Dai, Physikalisch-Technische Bundesanstalt (Germany)

Donglin Peng, Chongqing University of Technology (China)

3 Novel Instrument and Measurement System I

Kuang-chao Fan, National Taiwan University (Taiwan, China)

Ling Hao, National Physical Laboratory (United Kingdom)

$4 \quad$ Novel Instrument and Measurement System II

Igor A. Konyakhin, Saint-Petersburg State University of Information Technologies, Mechanics and Optics (Russia)

Qibo Feng, Beijing Jiaotong University (China)

$5 \quad$ Modern Optics and Instruments for Precision Measurement I

Jun Yang, National University of Defense Technology (China)

Liang-Chia Chen, National Taipei University of Technology

(Taiwan, China) 
6 Sensors, Actuators and Application I

Martin Booth, University of Oxford (United Kingdom)

Zi Xue, National Institute of Metrology (China)

$7 \quad$ Micro and Nano Metrology, Macro Metrology

Ying Xu, China University of Petroleum (China)

Xiaodong Wang, Dalian University of Technology (China)

8 Laser Measurement Techniques and Instruments I

Ahmed Abou-Zeid, Physikalisch-Technische Bundesanstalt (Germany)

Weihu Zhou, Academy of Opto-electronics, Chinese Academy of Sciences (China)

9 Instrumentation Theory and Methodology II

Frank Härtig, Physikalisch-Technische Bundesanstalt (Germany)

Zhaoyao Shi, Beijing University of Technology (China)

10 Instrument and Measurement System Calibration I

Baoli Yao, Xi'an Institute of Optics and Precision Mechanics, Chinese Academy of Sciences (China)

Liandong Yu, Hefei University of Technology (China)

11 Signal Processing and Image Processing

Yongying Yang, Zhejiang University (China)

Dengxin Hua, Xi'an University of Technology (China)

12 Sensors, Actuators and Application II

Ming Chang, Chung Yuan Christian University (Taiwan, China)

Yan Zhang, Capital Normal University (China)

13 Laser Measurement Techniques and Instruments II

Seung-Woo Kim, Korea Advanced Institute of Science and

Technology (Korea, Republic of)

Benyong Chen, Zhejiang Sci-Tech University (China)

$14 \quad$ Novel Instrument and Measurement System III

Sitian Gao, National Institute of Metrology (China)

Changcai Cui, Huagiao University (China)

15 Modern Optics and Instruments for Precision Measurement II

Richard Leach, National Physical Laboratory (United Kingdom)

Jian Liu, Harbin Institute of Technology (China)

16 Instrument and Measurement System Calibration II

Ling Hao, National Physical Laboratory (United Kingdom)

Peng Jin, Harbin Institute of Technology (China) 
Proc. of SPIE Vol. $9446944601-24$

Downloaded From: https://www.spiedigitallibrary.org/conference-proceedings-of-spie on 26 Apr 2023 Terms of Use: https://www.spiedigitallibrary.org/terms-of-use 


\section{Symposium Welcome}

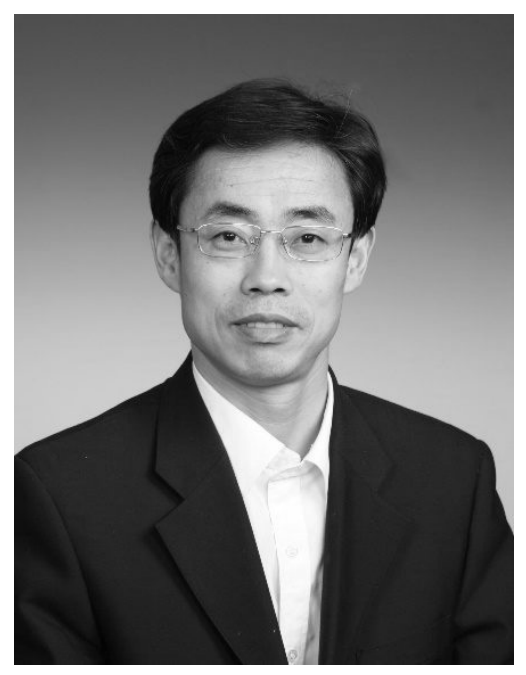

Ladies and Gentleman,

Good morning everybody!

While we are all gathering here today for ISPEMI 2014, I extend on behalf of the Chinese Society for Measurement warm congratulations to the convention of ISPEMI 2014.

Since its founding, the Instrumentation Committee has been promoting the development of measurement instrumentation industry, and the exchange of measurement technologies.

Somebody likened instrumentation to the eyes of human being, but I want to say the future instrumentation is no longer eyes only. It will have the function of brain and play the role of hands, and thus becomes really a "man". This "man" is a very clever man, too. He can recognize different things with different eyes (sensors) to generate different measurements. He can do different analyses with his brain, generate new instructions, and does different operations with his hands. Intelligentization, integration, modularization and accuracy enhancement are the future development trend of measurement instrumentation.

Low carbon economy, scientific approach to development, people's happiness is the focus of world attention, and the ultimate goals for measurement services as well. Monitoring the change of the earth, the sole home of mankind, customization production at future factories, energy efficiency and diversification of energy supply, healthy citizens, life with security guaranteed are all ultimate goal for our future development. We have a lot to do in the future, and we have a wide prospect for future development.

In recent years, many countries worked out development plans for development of measurement instrumentation, and reinforced investment into its development. It can be seen from The Measurement Instrumentation Development Plan for 2013-2020 published by the State Council of the People's Republic of China in March of 2013, that innovation and breakthrough are the keys for the development of measurement technologies. So I sincerely hope we could work closely with our colleagues all over the world to achieve breakthrough and innovation in highly sophisticated techniques, development of core detail parts for instrumentation, autonomy intellectual property right and way of technical exchange, especially innovation in measurement concept. Let's provide better 
measurement service for the economic development.

I wish ISPEMI 2014 a complete success!

Thanks!

Mr. Aiwen Ma

Secretary-general of Chinese Society for Measurements, China

9 August 2014, Changsha, China

Ma Aiwen

xxvi

Proc. of SPIE Vol. $9446944601-26$

Downloaded From: https://www.spiedigitallibrary.org/conference-proceedings-of-spie on 26 Apr 2023 Terms of Use: https://www.spiedigitallibrary.org/terms-of-use 


\section{Introduction}

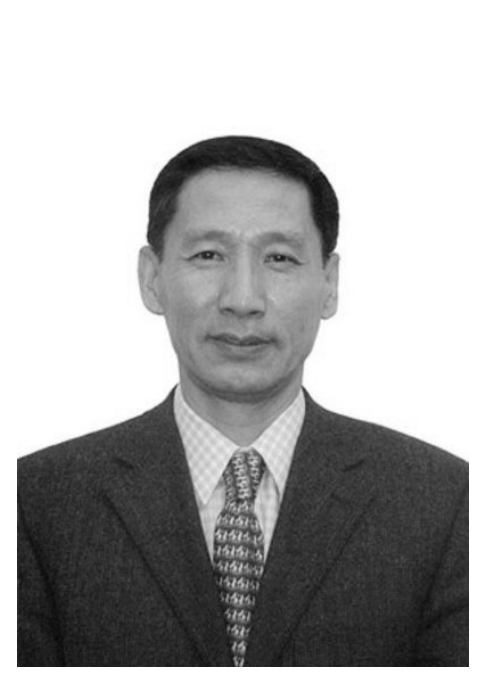

The International Symposium on Precision Engineering Measurements and Instrumentation (ISPEMI) is an international symposium held every other year in different cities of China with English as its working language. It originated from International Symposium on the Instrumentation Science and Technology (ISIST), which has been held for a total of 5 sessions. These meetings in series have greatly promoted the academic exchange in this particular field. In 2010, ISIST was converted to ISPEMI, and the use of ISPEMI means the establishment of a more efficient platform for the academic exchange among well-accomplished scientists and postgraduates both inside and outside China to facilitate the in-depth discussion and cooperation in and instrumentation. the field of precision engineering measurements

ISPEMI 2014 is the 9th ISPEMI, and was held 8-10 August 2014, in Changsha, Hunan Province. The keywords for the present meeting are "major frontier subjects of science and technology, overlapping and fusion of subjects, innovative instrumentation an ultra-precision engineering." More than 300 delegates and guests attended the meeting. Plenary speakers from the United States, United Kingdom, Australia, Japan, Germany, Russia, and China made excellent presentations on their work. A wide range of subjects was covered during session discussions. The meeting was an international technical forum for scientists, research workers and students working in the field of precision engineering measurements and instrumentation in different countries and regions to present their work, and to develop their knowledge of recent advances on this particular aspect.

From the 387 manuscripts received, we accepted only 225 papers for oral and poster presentation at the meeting. After the meeting, we worked further together with authors to make sure all the papers included in the proceedings of ISPEMI 2014 are good in both technical quality and English.

While the proceedings of ISPEMI 2014 is now ready for distribution to the authors, we would like to thank International Committee on Measurements and Instrumentation, National Natural Science Foundation of China, Chinese Society for Measurement, China Instrument and Control Society, Harbin Institute of Technology, National University of Defense Technology, Beijing Information Science and Technology University, and Hefei University of Technology for their funds and 
assistances provided. Our thanks go to the procedure and organizing committee members, especially honorary chairman, co-chairmen, and plenary speakers, Prof. Tony Wilson, Prof. Frank Härtig, Prof. Lihong V. Wang, Prof. Lars Montelius, Prof. Shuang Zhang, Prof. Saulius Juodkazis and Prof. Yiming Zhu for their efforts to make the meeting fruitful and successful. And our special thanks go to SPIE for its efforts to enable us to do all these so well.

Prof. Jiubin Tan Conference Chair of ISPEMI 2014

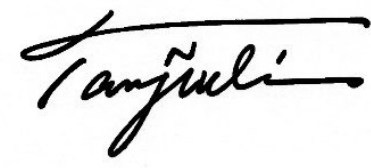

